\title{
Forearm fracture sequelae
}

\author{
Lorenzo Di Mento', Leda Staletti², Matteo Cavanna', \\ Marco Berlusconi ${ }^{1}$ \\ ${ }^{1}$ Department of Traumatology, Humanitas Research Hospital IRCCS, Milan, Italy; \\ ${ }^{2}$ Humanitas University at Humanitas Research Hospital IRCCS, Milan, Italy
}

Received: December 9, 2019

Accepted: January 14, 2020

\section{Correspondence}

\section{Lorenzo Di Mento}

Department of Traumatology, Humanitas Research Hospital IRCCS, via Manzoni 56, 20089 Rozzano (MI), Italy

Tel. +39028224 4547

E-mail: lorenzo.dimento@humanitas.it

Conflict of interest

The Authors declare no conflict of interest

How to cite this article: Di Mento L, Staletti L, Cavanna M, et al. Forearm fracture sequelae. Lo Scalpello 2020;34:38-41. https://doi. org/10.36149/0390-5276-005

(C) Ortopedici Traumatologi Ospedalieri d'Italia (O.T.O.D.I.) 2020

\section{(c) (i) (3) $(-)$}

This is an open access article distributed in accordance with the CC-BY-NC-ND (Creative Commons Attribution-NonCommercial-NoDerivatives 4.0 International) license. The article can be used by giving appropriate credit and mentioning the license, but only for non-commercial purposes and only in the original version. For further information: https://creativecommons.org/licenses/by-nc-nd/4.0/deed.en

\section{SUMMARY}

Forearm fractures are quite common at any age. When affecting children, they are mostly treated conservatively, since the remodeling potential is higher than adults and children can tolerate greater reduction defects. When affecting adults, the treatment is mostly surgical if the fracture shows a certain grade of displacement. The radio and ulna must be considered as an "extended joint" and for this reason their treatment follows the principles of "anatomical reduction" and "absolute stability", when possible, with an adequate technique to ensure early mobilization. If not properly treated, the outcome of these fractures is usually poor, due to non-union, mal-union or ossification of the interosseous membrane, with consequent pain, deformity and rigidity. We report six clinical cases.

Key words: forearm fractures, non union, delayed union, fracture sequelae, absolute stability

When treating forearm fractures, age is a crucial factor. In children, forearm fractures are mainly treated bloodlessly, and, when needed, through corrective maneuvers, because they have a greater remodelling potential than adults and tolerate greater reduction defects ${ }^{1-3}$.

We will focus about forearm fractures in adults, whose functional outcomes are disabling and easily predictable if not properly treated. Since the forearm behaves like a large joint whose main movement is prone-supination, an injury to the forearm can also affect the movement of the joints nearby wrist and elbow.

The possible outcomes of a forearm fracture that is not properly treated vary from delayed consolidation to malunion or healing with the formation of ossification in the interosseous membrane, with consequent pain, deformity and rigidity.

The main tendency is to treat a single-bone fracture in a conservative way. The immobilization must be kept on for minimum one month and the brace must include wrist and elbow joints in order to abolish the prone-supination, but the treatment does not always achieve the desired results.

We report six clinical cases below.

\section{Case 1}

Sixteen-year-old boy, isolated ulna fracture (Fig. 1A) treated conservatively with the immobilization in cast for 6 weeks (Fig. 1B). At first follow-up, X-rays showed a displacement of the ulnar fracture, which is not acceptable in the adult. After two months, the fracture line was still clearly visible and there was pain at the fracture site (Fig. 1C). Since the local biology seemed adequate, more stability was given through bridging plating, respecting the periosteum. At three months, the fracture healed (Fig. 1D). 


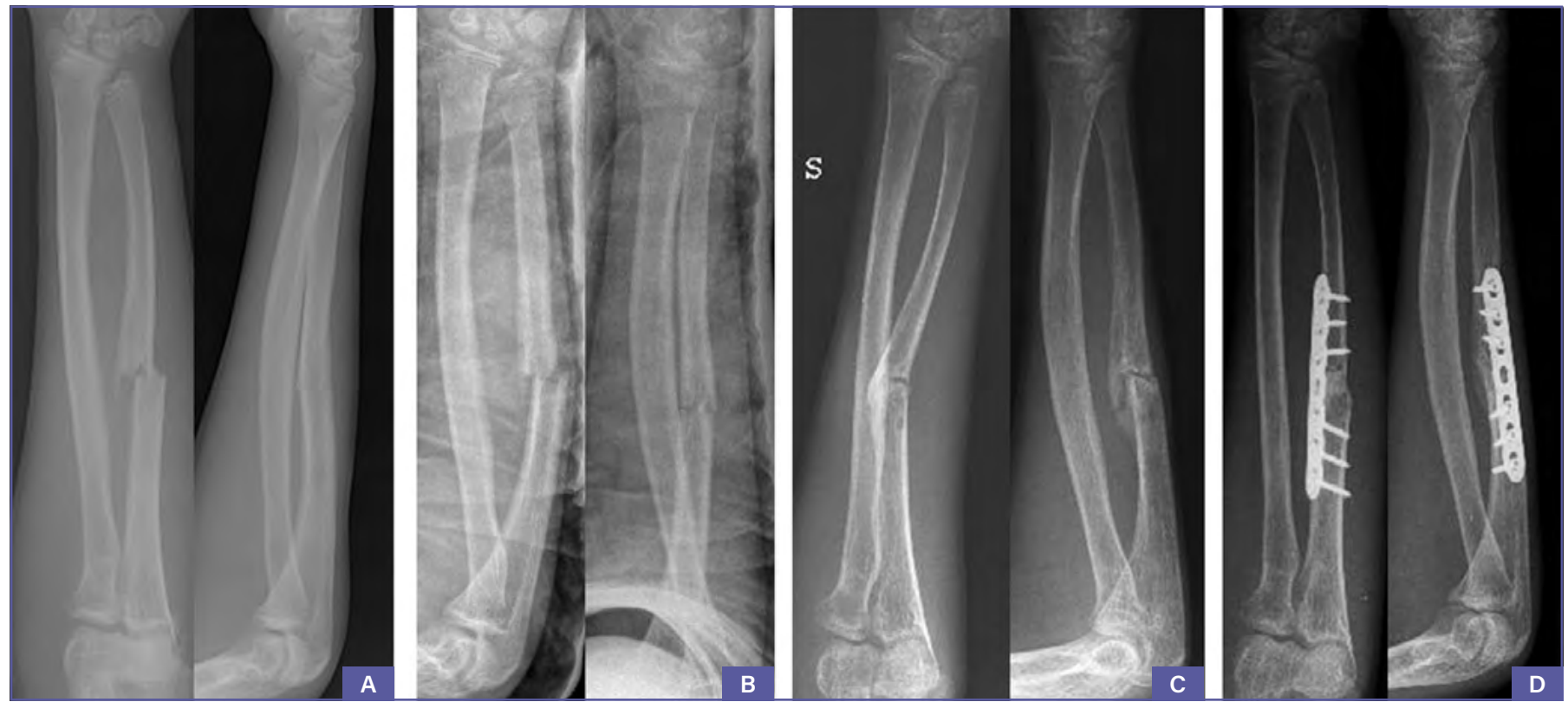

Figure 1. Delayed union due to lack of stability after conservative treatment for isolated ulna fracture: healing after plating.

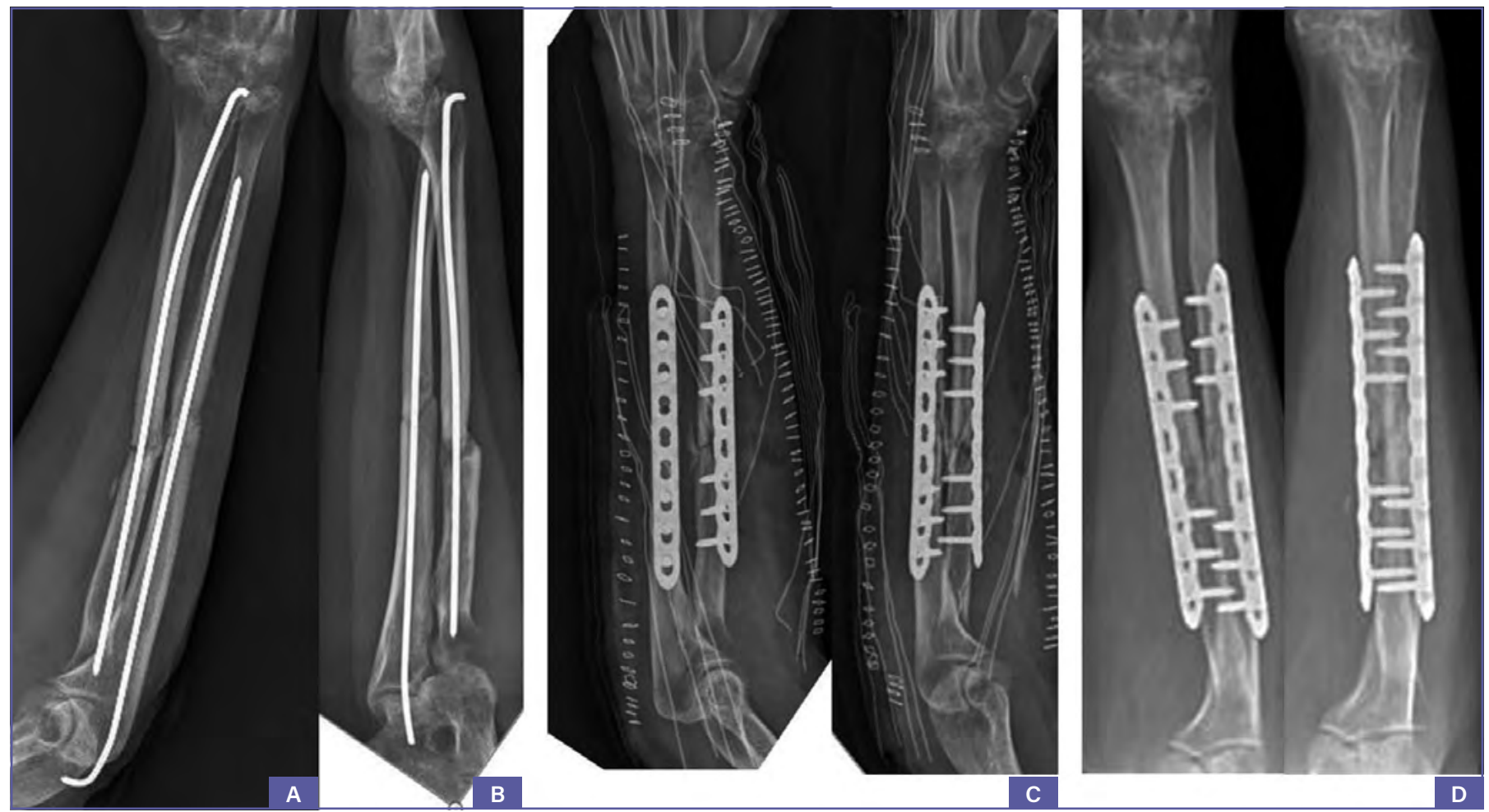

Figure 2. Non-union due to lack of stability after synthesis with TEN: healing after double plating. 


\section{Case 2}

Forty-year-old woman suffering from severe rheumatoid arthritis: the two-bones fracture of the forearm had been initially treated in another hospital with two TENs followed by three months of cast immobilization (Fig. 2A). The patient complained of pain and limited prone-supination. The immobilization must be congruous in order to have a functional recovery, which is always the main outcome to pursue, even through difficult surgery. Fracture was consequently stabilised by two bridging plates, without affecting or damaging the biology of the fracture site (Fig. 2B). We allowed immediate mobilization without restriction. At four months, the fracture healed (Fig. 2C).

\section{Case 3}

Forearm two-bones fracture (Fig. 3A) treated with double plating and subsequent immobilization with a cast. At three months, there was pain, deformity and delayed union (Fig. 3B). We decided to perform a new operation, through implant removal, decortication of the delayed-union site, renewal of the synthesis with double plating and autologous bone grafting (Fig. 3,C). At four months, the fracture healed (Fig. 3D).

\section{Case 4}

Sixty-year-old man, coming to our observation for pain and forearm deformity six months after an open reduction and internal fixation of a two-bones forearm fracture. X-rays showed an unbalanced synthesis with inadequate short plates (1/3 tubular plate) and screws lying in the fracture site (Fig. 4A). We performed implant removal, decortication, bone grafting from the iliac crest and renewal of the synthesis with double plating. At four months, the fracture healed (Fig 4B).

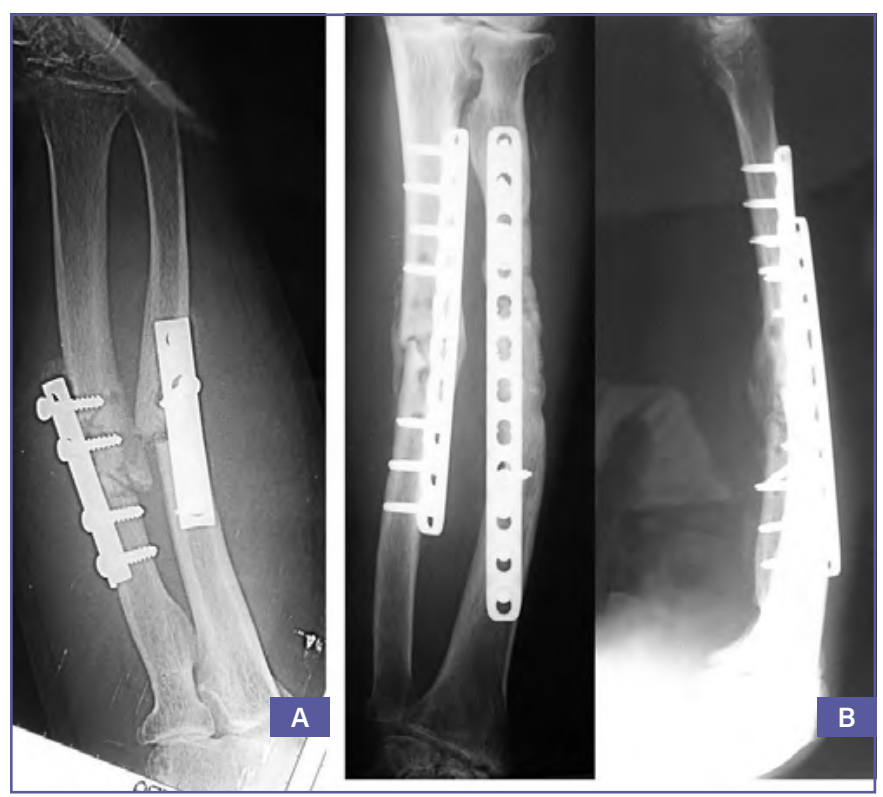

Figure 4. Nonunion after unbalanced synthesis with short plates: healing after re-plating and bone grafting.
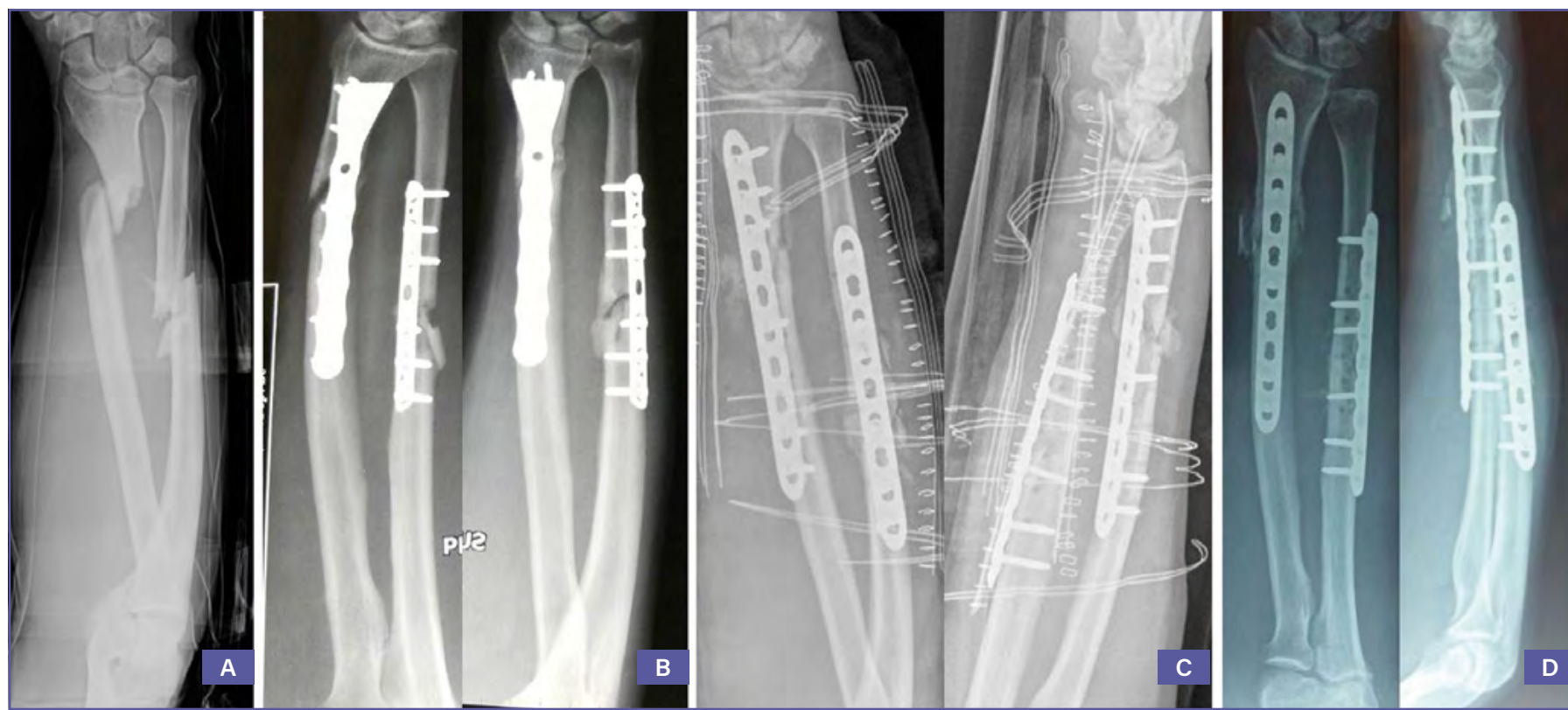

Figure 3. Delayed union after inadequate plating with gap and screws in fracture site: healing after re-plating and bone grafting. 


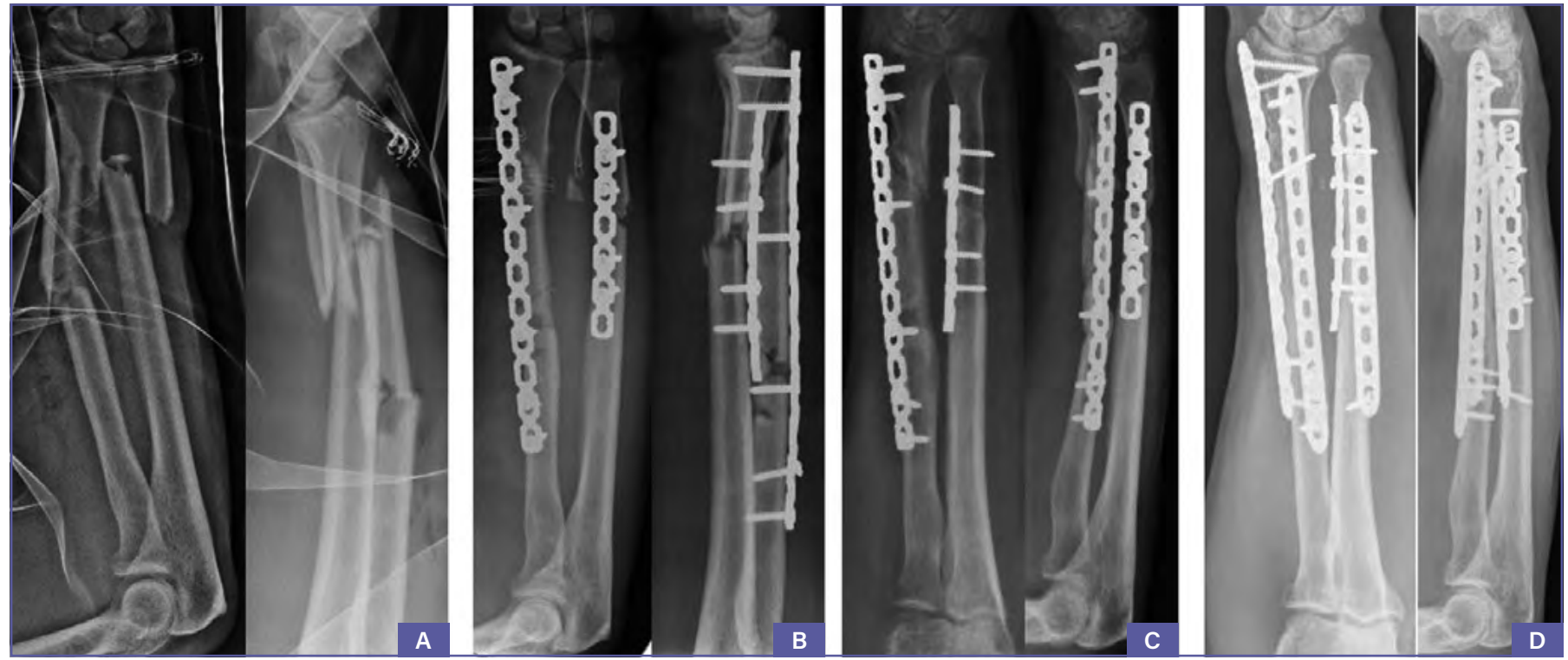

Figure 5. Nonunion after plating of open segmental fracture, with gap and mal-reduction: healing after re-plating and bone grafting.

\section{Case 5}

Forty-seven-year-old man, with open two-bones fracture (Fig. 5A) treated with double recon-type plating through a single surgical approach (the ulnar plate was placed dorsally through the exposition): the fracture was not anatomically reduced (X-rays showed a gap) and one screw lied in the fracture site (Fig. 5B). At 8 months, there was pain and slight deformity (Fig. 5C). We decided to renew the synthesis through a double surgical approach, with double plating on the radius and double plating plus bone grafting on the ulna, to achieve more stability. At three months, the fracture healed (Fig. 5D).

\section{Case 6}

Sixty-two-year-old man, coming to our observation 6 months after surgery with pain and deformity. X-rays showed an unbalanced synthesis, a malunion that resulted in a defect in the axis and a bone-loss in both bones. (Fig. 6A). During the revision surgery, after removing the internal fixation devices, $1.5 \mathrm{~cm}$ of non-vital bone was found in the ulna. We decided to reduce and compress the fracture (through the tension device) with autologous tricortical bone grafting from the iliac crest. On the radius we performed a cruentation and renewal of synthesis. At two months, X-rays showed signs of callus formation (Fig. 6B); at one year, the fracture healed (Fig. 6C).

In conclusion, we can state that the non-respect of the principles inevitably leads to failure. Forearm fractures are joint fractures and require absolute stability whenever possible with an adequate technique to ensure early mobilization ${ }^{4}$.
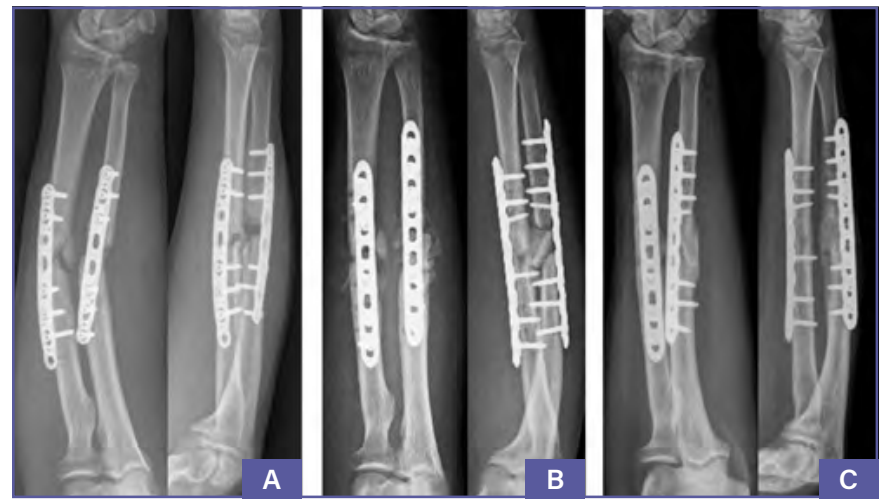

Figure 6. Nonunion after unbalanced synthesis and loss of reduction: healing after re-plating with compression and bone grafting.

\section{References}

Gladden PB, Wilson CH, Suk M. Pediatric orthopedic trauma: principles of management. Semin Pediatr Surg 2004;13:119-25. https://doi.org/10.1053/j.sempedsurg.2004.01.008

2 Boutis K. The emergency evaluation and management of pediatric extremity fractures. Emerg Med Clin North Am 2020;38:31-59. https://doi.org/10.1016/j.emc.2019.09.003

Lien J. Pediatric orthopedic injuries: evidence-based management in the emergency department. Pediatr Emerg Med Pract 2017;14:1-28.

4 Bukley RE, Moran CG, Apavatthakakul T. AO principles of fracture management. $3^{\text {rd }}$ ed., 2018. 